\title{
Impact of deleterious mutations, sexually antagonistic selection, and mode of recombination suppression on transitions between male and female heterogamety
}

\author{
Paul A. Saunders $\mathbb{1}^{1} \cdot$ Samuel Neuenschwander ${ }^{1,2} \cdot$ Nicolas Perrin $\mathbb{1}^{1}$ \\ Received: 25 January 2019 / Revised: 18 March 2019 / Accepted: 23 March 2019 / Published online: 27 April 2019 \\ (c) The Genetics Society 2019
}

\begin{abstract}
Deleterious mutations accumulating on non-recombining $\mathrm{Y}$ chromosomes can drive $\mathrm{XY}$ to $\mathrm{XY}$ turnovers, as they allow to replace the old mutation-loaded $\mathrm{Y}$ by a new mutation-free one. The same process is thought to prevent $\mathrm{XY}$ to $\mathrm{ZW}$ turnovers, because the latter requires fixation of the ancestral $\mathrm{Y}$, assuming dominance of the emergent feminizing mutation. Using individual-based simulations, we explored whether and how an epistatically dominant $\mathrm{W}$ allele can spread in a young XY system that gradually accumulates deleterious mutations. We also investigated how sexually antagonistic (SA) polymorphism on the ancestral sex chromosomes and the mechanism controlling X-Y recombination suppression affect these transitions. In contrast with $\mathrm{XY}$ to $\mathrm{XY}$ turnovers, $\mathrm{XY}$ to $\mathrm{ZW}$ turnovers cannot be favored by $\mathrm{Y}$ chromosome mutation load. If the arrest of $\mathrm{X}-\mathrm{Y}$ recombination depends on genotypic sex, transitions are strongly hindered by deleterious mutations, and totally suppressed by very small SA cost, because deleterious mutations and female-detrimental SA alleles would have to fix with the $Y$. If, however, the arrest of $X-Y$ recombination depends on phenotypic sex, $X$ and $Y$ recombine in XY ZW females, allowing for the purge of Y-linked deleterious mutations and loss of the SA polymorphism, causing XY to $\mathrm{ZW}$ turnovers to occur at the same rate as in the absence of deleterious and sex-antagonistic mutations. We generalize our results to other types of turnovers (e.g., triggered by non-dominant sex-determining mutations) and discuss their empirical relevance.
\end{abstract}

\section{Introduction}

Several lineages of plants and animals (including birds, mammals, and Drosophila) present highly differentiated sex chromosomes, where a large, gene-rich $\mathrm{X}$ (or Z) chromosome contrasts with a small, gene-poor, and degenerated $\mathrm{Y}$ (or W) chromosome. The canonical model of sexchromosome evolution assigns a crucial role to sexually antagonistic (SA) mutations to the first step of the process

Supplementary information The online version of this article (https:// doi.org/10.1038/s41437-019-0225-z) contains supplementary material, which is available to authorized users.

Paul A. Saunders

paul.alan.saunders@gmail.com

1 Department of Ecology and Evolution, University of Lausanne, 1015 Lausanne, Switzerland

2 Vital-IT, Swiss Institute of Bioinformatics, University of Lausanne, 1015 Lausanne, Switzerland
(Fisher 1931; Charlesworth and Charlesworth 1980; Rice 1984): in the case of a young XY system, a male-beneficial mutation occurring on the proto-Y chromosome should spread, even if highly detrimental to females, because it is more likely to be transmitted to sons than to daughters. As a second step, recombination will be suppressed between this SA locus and the sex-determining locus (e.g., through an inversion), generating strict co-segregation of the two loci (Rice 1987). As a side effect, however, recombination arrest will induce the accumulation of deleterious mutations on the $\mathrm{Y}$ chromosome, followed by gene loss and degeneration, and possibly accompanied by dosage compensation (Bergero and Charlesworth 2009).

However, many other lineages (including in teleost fish, amphibians, and non-avian reptiles) present homomorphic sex chromosomes. Two main reasons have been invoked to account for this lack of differentiation. First, if genetic control over sex determination is not complete (allowing for occasional sex-reversal), and recombination arrest depends on phenotypic sex, then $\mathrm{X}$ and $\mathrm{Y}$ chromosomes will recombine occasionally in sex-reversed $\mathrm{XY}$ females, 
preventing sex-chromosome differentiation in the long-term (the 'fountain-of-youth' model; Perrin 2009; Rodrigues et al. 2018). Second, sex chromosomes in these lineages might show high rates of turnovers, whereby a new pair replaces the ancestral sex chromosomes before they have had time to degenerate (Schartl 2004; Volff et al. 2007). Several processes may drive such turnovers. Bull and Charnov (1977) proposed over four decades ago that turnovers could be mediated by genetic drift. So-called neutral turnovers, when driven by sex-determining mutations that are epistatically dominant (i.e., that override the action of the resident sex-determining gene), are more likely to occur than substitutions at neutral autosomal loci, and turnovers that induce a change in the heterogamety pattern (e.g., transition from an XY system to a ZW system) tend to become more likely as effective population size $\left(N_{\mathrm{e}}\right)$ decreases (Veller et al. 2017). Nevertheless, neutral turnovers that maintain the system of heterogamety (e.g., XY to a new XY system) are 2-4 times more likely to occur than the former (except under extremely low $N_{\mathrm{e}}$; Saunders et al. 2018). One reason is that an $X Y$ to $X Y$ turnover requires the fixation of the ancestral $X$ chromosome as an autosome while an $\mathrm{XY}$ to $\mathrm{ZW}$ turnover requires the fixation of the ancestral Y (when driven by epistatically dominant mutations). The initial difference in frequency between the $\mathrm{X}$ and the $\mathrm{Y}(0.75$ vs. 0.25$)$, makes the fixation of the $\mathrm{X}$ chromosome by chance more likely. A second potential driver for turnovers is SA selection: if a mutant sex determiner arises in close linkage with an autosomal SA allele beneficial for the sex determined by that mutation (e.g., mutant male determiner in close linkage with a male-beneficial allele), that newly arisen determiner will have a selective advantage, as the male beneficial-female detrimental allele will be more likely to be inherited by sons than daughters. SA selection was shown to be able to facilitate both transitions that maintain the heterogamety pattern, and transitions that change it (van Doorn and Kirkpatrick 2007, 2010), as potentially illustrated by the XY to ZW transition documented in cichlid fishes (Roberts et al. 2009). A third potential driver of turnovers is the load of deleterious mutations on non-recombining $\mathrm{Y}$ (or W) chromosomes. Blaser et al. $(2013,2014)$ formalized this model for an $\mathrm{XY}$ to $\mathrm{XY}$ transition, and showed that, given specific combinations for the coefficients of selection $(s)$ and dominance $(h)$ of deleterious mutations accumulating on the $\mathrm{Y}$, as well as effective population size $N_{\mathrm{e}}$, the benefits of fixing a new, mutation-free $Y$ chromosome can outweigh the cost of losing male-beneficial mutations fixed on the ancestral $\mathrm{Y}$ chromosome. Mutation-load driven transitions have been proposed to account for the high rate of XY to XY turnover documented in ranid frogs (Jeffries et al. 2018). This mechanism has indeed been suggested to hinder XY to ZW transitions, because the ancestral $\mathrm{Y}$ fixes as an autosome once the transition is over, which is obviously detrimental if it is loaded with deleterious mutations (Blaser et al. 2013, 2014). However, theoretical evidence has been provided only for cases where deleterious mutations have a strong negative effect on fitness, and are completely recessive (van Doorn and Kirkpatrick 2010; Veller et al. 2017). While this situation is well suited to account for systems in which the degeneration of the $\mathrm{Y}$ has advanced to the point where YY individuals bear large fitness costs, it is less applicable to young sex-chromosome systems, which have had less time to accumulate deleterious mutations.

In this paper, we model the evolution of young nonrecombining sex chromosomes (XY), which gradually accumulate deleterious mutations and carry a SA locus. We use individual-based simulations to explore quantitatively whether and how different values of $h, s$, and $N_{\mathrm{e}}$ effectively prevent XY to ZW turnovers driven by epistatically dominant autosomal $\mathrm{W}$ mutations. Additionally, we explore whether and how this outcome depends on the mechanism arresting recombination between the sex chromosomes. This arrest can result from a sex-specific recombination suppression genome-wide, as documented in Drosophila (male achiasmy; Morgan 1914) and some Lepidoptera (female achiasmy; Tanaka 1914). Note that recombination is not necessarily entirely suppressed in the heterogametic sex: in some amphibians, including the European tree frogs Hyla arborea and the common frog Rana temporaria, male recombination is restricted to the tips of chromosomes, and largely absent in their center (Rodrigues et al. 2013; Brelsford et al. 2016; Jeffries et al. 2018). Alternatively, suppression can be specific to the sex-chromosome pair: caused by a chromosomal rearrangement (e.g., an inversion; Kirkpatrick 2010), or a gradual spread of the non-recombining region (e.g., through a change of the location of cross-over events; Charlesworth et al. 2005). A profound difference between the two modes of recombination suppression (genome-wide or chromosome specific) is that in the former, recombination between the two sex chromosomes can occur in sex-reversed XY females (e.g., Rodrigues et al. 2018), which might affect $X Y$ to $Z W$ turnovers as it raises the possibility of purging the $\mathrm{Y}$ in $\mathrm{XY} \mathrm{ZW}$ females.

\section{Methods}

For all simulations, we used a genetic model and chromosomal architecture similar to those in Blaser et al. (2013), and identical parameter values, which allowed us to directly compare the effect of $\mathrm{Y}$ chromosome mutation load on the dynamics of sex-chromosome turnovers that either change the pattern of heterogamety, or preserve it. 


\section{Genetic model}

\section{Sex determination}

The genomic model consists of one pair of sex chromosomes and one pair of autosomes (Fig. 1). The sex chromosomes (X and $\mathrm{Y}$ ) harbor the resident sex-determining locus with two alleles, $X$ and $Y$. Sex determination is strictly genetic, so that $X X$ individuals are females and $X Y$ individuals are males. The autosomal pair harbors a gene involved in the sexdetermination cascade, initially fixed for an allele $f$. This $f$ allele can mutate (unidirectionally) to a feminizing allele $F$ that overrides the masculinizing effect of allele $Y$ (epistatic dominance), so that $X Y f F$ individuals develop as females. If both loci are polymorphic, males can be $X Y$ ff or $Y Y$ ff, and females $X X f f, X X f F, X Y f F$, or $Y Y f F$ (see Table 1). A sexchromosome turnover is achieved once polymorphism at the ancestral sex-determining locus is lost, with all males being $Y Y f f$ and all females $Y Y f F$ (i.e., $\mathrm{ZZ}$ and $\mathrm{ZW}$ ).

\section{Deleterious mutations}

Both pairs of chromosomes carry ten functional genes evenly spaced $(10 \mathrm{cM})$ around the sex-determining loci (Fig. 1), which can mutate unidirectionally to a deleterious form. The effect on fitness of a single deleterious mutation depends on a selection coefficient $s$ and a dominance coefficient $h$, so that the fitness of an individual heterozygous for that locus is reduced by $h s$ and that of an individual homozygous for the mutant allele by $s$. Deleterious mutations affect the mutation load component of fitness $\left(w_{\mathrm{ML}}\right)$, which is assumed to be:

$w_{\mathrm{ML}}=(1-s)^{n_{\mathrm{hom}}} *(1-h s)^{n_{\mathrm{het}}}$

where $n_{\text {hom }}$ and $n_{\text {het }}$ are, respectively, the number of loci homozygous and heterozygous for a deleterious mutation.

\section{SA selection}

In some of our simulations, the sex-chromosome pair carries a bi-allelic sexually antagonistic locus (e.g., a coloration gene), inserted between the sex-determining locus and one of the two adjacent loci subject to deleterious mutation (genetic distance: $10 \mathrm{cM}$, Fig. 1). The two alleles are a male beneficial-female detrimental allele $\left(a^{\mathrm{m}}\right)$, and a female beneficial-male detrimental allele $\left(a^{f}\right)$. The sexually antagonistic component of fitness $\left(w_{\mathrm{SA}}\right)$, depends on the phenotypic sex and genotype of individuals at that locus: $w_{\mathrm{SA}}=1$ in $a^{\mathrm{m}} a^{\mathrm{m}}$ males and $a^{\mathrm{f}} a^{\mathrm{f}}$ females, $w_{\mathrm{SA}}=1-2 c$ in $a^{\mathrm{f}} a^{\mathrm{f}}$ males and $a^{\mathrm{m}} a^{\mathrm{m}}$ females and $w_{\mathrm{SA}}=1-c$ in all $a^{\mathrm{f}} a^{\mathrm{m}}$ heterozygotes, where $c$ is the cost of SA selection (i.e., the impact on fitness of bearing a detrimental allele).

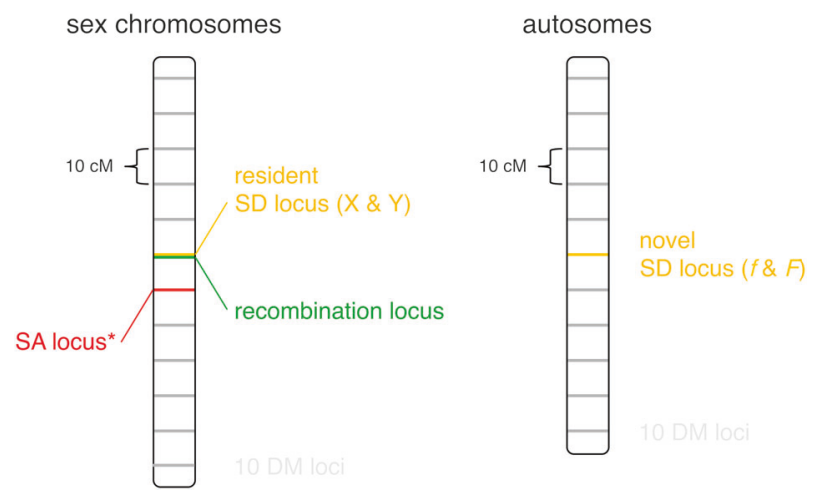

Fig. 1 Genetic architecture of the two chromosome pairs. Genetic distances shown are assuming loci are not in a non-recombining region or individual. SD: sex-determining, SA: sexually antagonistic, DM: deleterious mutation. The SA locus (see asterisk) was not present in the first three sets of simulations: the genetic distance between the SD locus and closest DM locus underneath it was $10 \mathrm{cM}$ in these simulations

Table 1 Genotypes present during a XY to ZW turnover, and presence or absence of $\mathrm{X}-\mathrm{Y}$ recombination for each genotype depending on mode of recombination suppression

\begin{tabular}{llll}
\hline \multirow{2}{*}{ Genotype } & Sex & \multicolumn{2}{l}{ Recombination depends on } \\
\cline { 3 - 4 }$X X f f$ & Penotypic sex & Genotypic sex \\
$X Y f f$ & Male & No & Yes \\
$X X f F$ & Female & Yes & No \\
$X Y f F$ & Female & Yes & Yes \\
$Y Y f f$ & Male & No & No \\
$Y Y f F$ & Female & Yes & Yes \\
\hline
\end{tabular}

In the presence of this locus, the total fitness of an individual $(W)$ is the product of its two components:

$$
W=w_{\mathrm{SA}} * w_{\mathrm{ML}}
$$

\section{Recombination pattern}

Recombination depends on either phenotypic sex or genotypic sex. In the first case, recombination occurs in females and never in males, regardless of their genotype. This mimics species in which recombination is either arrested or largely restricted genome-wide in the heterogametic sex (e.g., frogs, Drosophila). In the second case, all loci on the $\mathrm{Y}$ chromosome are assumed to be included in an inversion, so that sex-chromosome recombination occurs only in individuals homozygous at the ancestral sex-determining locus $(X X$ and $Y Y)$, regardless of phenotypic sex (Table 1). In practice, we implemented a bi-allelic locus controlling recombination rate. It is strictly linked to the $X Y$ sexdetermining locus in all individuals $(0 \mathrm{cM}$, Fig. 1$)$, and 
allows recombination either only in females regardless of genotypic sex, or only in homozygotes regardless of phenotypic sex. Recombination occurs without interference, at a rate $(r)$ that depends on the genetic distance in cM $(m)$ following Haldane's mapping function (Haldane 1919):

$r=\frac{1-e^{-2 m / 100}}{2}$

Therefore, $r \approx 0.09$ for two loci separated by $10 \mathrm{cM}$, and 0 in individuals in which recombination is not allowed.

\section{Simulations}

Individual-based simulations were run with quantiNemo 2 (Neuenschwander et al. 2018). All simulations were run for $10^{5}$ generations, and unless mentioned otherwise effective population size was set to $N_{\mathrm{e}}=10^{3}$. At each generation (non-overlapping), gametes were drawn with a probability proportional to individual fitness $W$, and gametes were paired randomly (one male and one female) to constitute the $N_{\mathrm{e}}$ individuals of the next generation (soft selection). The initial frequency of $X$ and $Y$ alleles on the sex-chromosome pair was set to 0.75 and 0.25 , respectively, and sex chromosomes were transmitted with equal probability (binomial distribution with probability 0.5), allowing for small fluctuations around an otherwise balanced sex ratio. On the autosomal pair, the sex-determining locus was initially fixed for the $f$ allele, and allowed to mutate to its dominant form $F$ at a rate $\mu_{\mathrm{SD}}=10^{-5}$. The loci subject to deleterious mutation were allowed to mutate to their deleterious form at a rate $\mu_{\mathrm{DM}}=10^{-4}$. The coefficients of deleterious mutations varied independently between sets of simulations (with values $s=\{0.001,0.0025,0.005,0.01,0.02,0.03,0.04$, $0.05,0.1,0.15,0.2\}$ and $h=\{0,0.1,0.15,0.25,0.5,0.75$, $1\})$. In a single run, $h$ and $s$ were the same for all loci. In models with sexually antagonistic selection, the $a^{\mathrm{m}}$ allele was initially fixed on the Y chromosome (frequency: 0.25) and the $a^{f}$ allele fixed on the $\mathrm{X}(0.75)$, and the cost $c$ took one of the two values $\{0.005,0.02\}$.

Five sets of simulations with different simulation regimes were run. A preliminary set of simulation was run to evaluate the fate of deleterious mutations on the $\mathrm{X}$ and the $\mathrm{Y}$ in the absence of turnovers $\left(\mu_{\mathrm{SD}}=0\right)$, and of SA selection (SA locus absent, see Fig. 1). For each $h \mathrm{x} s$ combination, we assessed how many deleterious mutations had gone to fixation on each chromosome by the end of the simulations (across 100 replicates). In the second set of simulations, we evaluated how the mode of recombination suppression affects turnovers, by assessing for each $h \times s$ combination (SA locus absent), the proportion of simulations in which a transition had occurred at the end of the $10^{5}$ generations (across 100 replicates). We also assessed the mean time to transition, which is the sum of the waiting time until a mutation $F$ appears and time for its "fixation" (note that several mutations might have appeared within the time needed for a transition, and that, because the mutation rate was chosen arbitrarily, we were interested in relative time to turnover rather than its absolute value). In the third set of simulations, we changed the value of $N_{\mathrm{e}}$ to $10^{2}$ and $10^{4}$, in order to evaluate the effect of effective population size on these turnovers. As $N_{\mathrm{e}}$ affects both the effect of drift and the availability of mutations, to evaluate the sole effect of drift on the outcome of our simulations, we also performed simulations with $N_{\mathrm{e}}=10^{2}$ and mutation availability identical to our previous models $\left(\mu_{\mathrm{SD}}=10^{-4}\right)$. A fourth set of simulations aimed at determining the impact of a SA gene located on the resident sex chromosomes on the dynamics of $\mathrm{XY}$ to $\mathrm{ZW}$ transitions in the presence of deleterious mutations. The SA locus was therefore inserted close to the SD locus (Fig. 1). First, we ran simulations with $c=\{0.005$, $0.02\}$ across the whole range of $h \times s$ combinations. As there was no evidence for an interaction between $h s$ and $c$ values in these simulations (Fig. 2), we limited further simulations (aimed at investigating more thoroughly the interaction between SA selection and recombination pattern on turnovers) to a smaller set of parameters: $h$ and $s$ values were set to 0 (neutral mutations), and $c$ varied from 0 to 0.02 with a 0.0025 increment. In case of recombination depending on phenotypic sex, we also explored a larger distance between the SD and SA loci (100 cM, loose linkage; $r \approx 0.43$; see Eq. 1). For this set of simulations, the proportion and mean time to transition were assessed, as well as the proportion and mean time to polymorphism loss at the SA locus. Finally, to check the full consistency of our simulations with those from Blaser et al. (2013), we ran a fifth set of simulation, replacing the feminizing allele $F$ by a masculinizing $M$ allele and replicated their simulations. Additionally, we also ran simulations with the $M$ allele across the whole range of $c$ values (with the 0.0025 increment), with either $h=s=0$ (no deleterious mutations), or $h=0.15$ and $s=0.02$ (so that $h s=0.003$; value that maximizes the positive impact of mutation load on $\mathrm{XY}$ to $\mathrm{XY}$ turnovers; Blaser et al. 2013).

\section{Results}

\section{The fate of deleterious mutations on sex chromosomes}

As expected, the fate of deleterious mutations depends on effective population size, as well as dominance and selection coefficients: mutations tend to go to fixation at low $N_{\mathrm{e}} h s$ values and are eliminated at high $N_{\mathrm{e}} h s$ values. As also found by Blaser et al. (2013), the shift between these two outcomes occurs between $\log _{10}\left(N_{\mathrm{e}} h s\right)=0$ and 1 in the non- 
Fig. 2 Proportion of replicates (across 100) in which an XY to $\mathrm{ZW}$ turnover has occurred at $T=10^{5}$ generations, as a function of $\log _{10}\left(N_{\mathrm{e}} h s\right)$ for $h$ from 0.1 to 1 (black to gray scale), or $\log _{10}\left(N_{\mathrm{e}} s\right)$ for $h=0$ (yellow), for different strengths of sexually antagonistic selection $(c=0,0.005$, and 0.02 ), and $N_{\mathrm{e}}=1000$. The vertical dashed line shows the threshold below which the Y starts accumulating deleterious mutations $\left(\log _{10}\left(N_{\mathrm{e}} h s\right)=1\right)$. The red horizontal dashed line shows turnover proportion (across 100 replicates) for a neutral case $(h=s=c=0)$
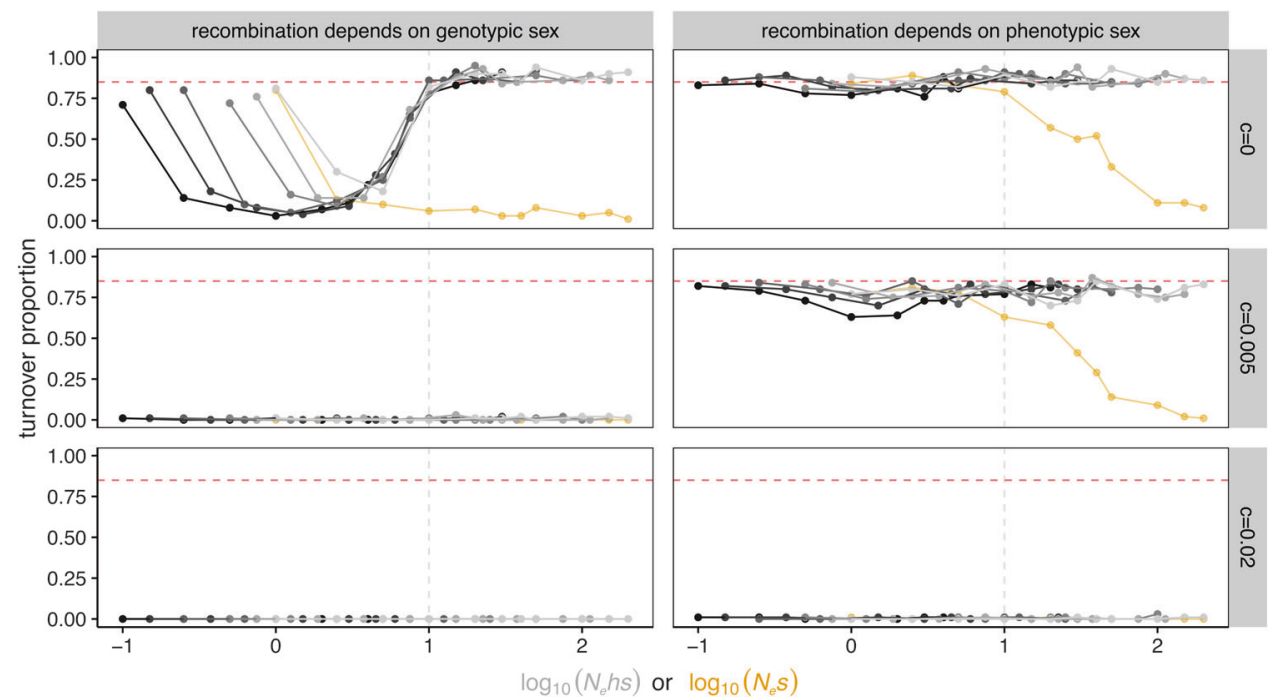

recombining region of the Y chromosome (Supplementary Fig. S1). Furthermore, we show that it occurs between $\log _{10}$ $\left(N_{\mathrm{e}} s\right)=0$ and 1 (i.e., independent of dominance) on the $\mathrm{X}$ chromosome (Supplementary Fig. S2). Note that fully recessive mutations $(h=0)$ can fix at higher $N_{\mathrm{e}} S$ values (Supplementary Fig. S1, yellow line), as they are relatively sheltered from selection, because purifying selection maintains these mutations at a low frequency on the $\mathrm{X}$ chromosome (Supplementary Fig. S2, yellow line). The two boundaries $\log _{10}\left(N_{\mathrm{e}} h s\right)=1$ and $\log _{10}\left(N_{\mathrm{e}} s\right)=0$ (defined above) delimit a window over which mutations accumulate on the $\mathrm{Y}$ more than on the $\mathrm{X}$, generating a selective pressure expected to affect the probability of turnovers. The range across which mutations have an effect too strong to accumulate even on the $\mathrm{Y}\left(\log _{10}\left(N_{\mathrm{e}} h s\right)>1\right)$ should provide, in the absence of SA selection $(c=0)$, a neutral expectation for the probability and mean time to sex-chromosome turnover, and a reference point to define whether other parameter combinations tend to favor or hinder turnovers.

\section{Impact of the mode of recombination arrest on turnovers}

Across the neutral range $\left(\log _{10}\left(N_{\mathrm{e}} h s\right)>1\right)$, turnover probability in our first set of simulations $\left(N_{\mathrm{e}}=10^{3}, c=0\right)$ averaged $\sim 0.85$ after $10^{5}$ generations, for any positive $h$ value (Fig. 2 top panels, right of the dashed vertical line), with a mean time to turnover close to 35,000 generations (Supplementary Fig. S3). This did indeed conform to the neutral expectation ( $s=h=0$, red horizontal lines on Fig. 2 and Supplementary Fig. S3). Fully recessive mutations ( $h$ $=0)$, on the other hand, drastically hindered turnovers at any $s$ value. Outside of this range $\left(\log _{10}\left(N_{\mathrm{e}} h s\right)<1\right)$, the outcomes of simulations strongly depended on the mechanism of recombination suppression. When recombination was dependent on genotypic sex, on the one hand, turnover probability dropped sharply below $\log _{10}$ $\left(N_{\mathrm{e}} h s\right)=1$ (Fig. 2 top left panel, left of the dashed vertical line), meaning that the load of deleterious mutations strongly hindered $\mathrm{XY}$ to $\mathrm{ZW}$ turnovers. The mean time to turnover similarly dropped (Supplementary Fig. S3), showing that $\mathrm{XY}$ to $\mathrm{ZW}$ turnovers are progressively prevented as deleterious mutations accumulate on the $\mathrm{Y}$ (i.e., turnovers are more likely in young sex-chromosome systems, that had less time to accumulate deleterious mutations). Probabilities and time to turnover were higher for small $s$ values, for which mutations also accumulate on the $\mathrm{X}\left(s=0.001\right.$, i.e., $N_{\mathrm{e}} s=1$, regardless of $\left.h\right)$. When recombination was dependent on phenotypic sex, on the other hand, deleterious mutations had no visible effect on the likelihood of turnover (Fig. 2, top right panel) and time to turnover (Supplementary Fig. S3), unless mutations were fully recessive (in which case transition probability decreased with increasing values of $s$ ). The reason the mode of recombination arrest has such a strong impact on turnover probability is that the emergence of a feminizing mutation allows the $\mathrm{Y}$ chromosome to be regularly purged through recombination with the $\mathrm{X}$ chromosome whenever $X Y f F$ females appear in the population, but only if recombination depends on phenotypic sex (Supplementary Fig. S4).

\section{Impact of effective population size}

Varying effective population size had no qualitative effect on turnovers when recombination was dependent on genotypic sex within the parameter spaced tested (Supplementary Figs. S5 and S6): regardless of $N_{\mathrm{e}}$, the likelihood and mean time to turnover dropped at $h s$ values that allow deleterious mutations to accumulate on the $\mathrm{Y}$ but not on the $\mathrm{X}$ (i.e., 
between $\log _{10}\left(N_{\mathrm{e}} h s\right)=1$ and $\left.\log _{10}\left(N_{\mathrm{e}} s\right)=0\right)$. In contrast, $N_{\mathrm{e}}$ had a qualitative effect when recombination was dependent on phenotypic sex. In populations with large effective size $\left(N_{\mathrm{e}}=10^{4}\right)$, even fully recessive mutations $(h=0)$ had no effect on transition likelihood; while in smaller ones $\left(N_{\mathrm{e}}=\right.$ $10^{2}$ ), a drop also occurred at intermediate $h s$ values (strongest effect around $\log _{10}\left(N_{\mathrm{e}} h s\right)=0$, Supplementary Fig. S5), but with no effect on the mean time to turnover (Supplementary Fig. S6). The reason is that purifying selection is less efficient at keeping deleterious mutations at a low frequency on the $\mathrm{X}$ chromosome in populations with smaller effective size (Supplementary Fig. S2), so the Y chromosome cannot be purged as efficiently in sex-reversed females. In the additional simulations with $N_{\mathrm{e}}=10^{2}$ and $\mu_{\mathrm{SD}}=10^{-4}$ (in which the availability of mutations was equivalent to that of our baseline models), no qualitative difference was observed (Supplementary Fig. S7).

\section{Turnovers in presence of a sexually antagonistic locus}

The effect of sexually antagonistic selection also varied along with the mode of recombination arrest. When recombination depended on genotypic sex, turnovers were totally prevented by even very weak SA selection (Fig. 2 and Supplementary Fig. S3, middle and bottom left panels). When recombination depended on phenotypic sex, weak SA selection ( $c=0.005$; Fig. 2 and Supplementary Fig. S3, middle right panels) had little effect on the likelihood of turnover events, or time until a turnover occurred, compared to simulations without SA selection $(c=0)$ : over all $h s$ values $>0$, the turnover probability dropped only from $0.85 \pm 0.04$ (mean $\pm \mathrm{sd}$ ) to $0.78 \pm 0.05$, though stronger SA selection ( $c=0.02$; Fig. 2, lower right panel) completely hindered turnovers.

Broadening the values for $c$ showed that, as expected, turnover probability gradually decreases with the strength of SA selection (Fig. 3, black lines), and confirmed that the negative effect of SA selection on turnovers is much stronger when recombination suppression depends on genotypic sex rather than phenotypic sex. In the latter case, increasing the genetic distance between the sex-determining locus and the SA locus (from 10 to $100 \mathrm{cM}$, i.e., increasing recombination rate from 0.09 to 0.43 ) further increased turnover likelihood and decreased mean time to turnover (Fig. 3 and Supplementary Fig. S8, black lines), suggesting that recombination between the two loci is what reduces the negative effect of SA selection on transitions from male to female heterogamety.

Analyzing the allelic composition of the SA locus at the end of simulations revealed that the probability that one of the two SA alleles goes to fixation also decreases with the strength of SA selection $c$ (Fig. 3, red lines) while the mean time to fixation increases (Supplementary Fig. S8). These

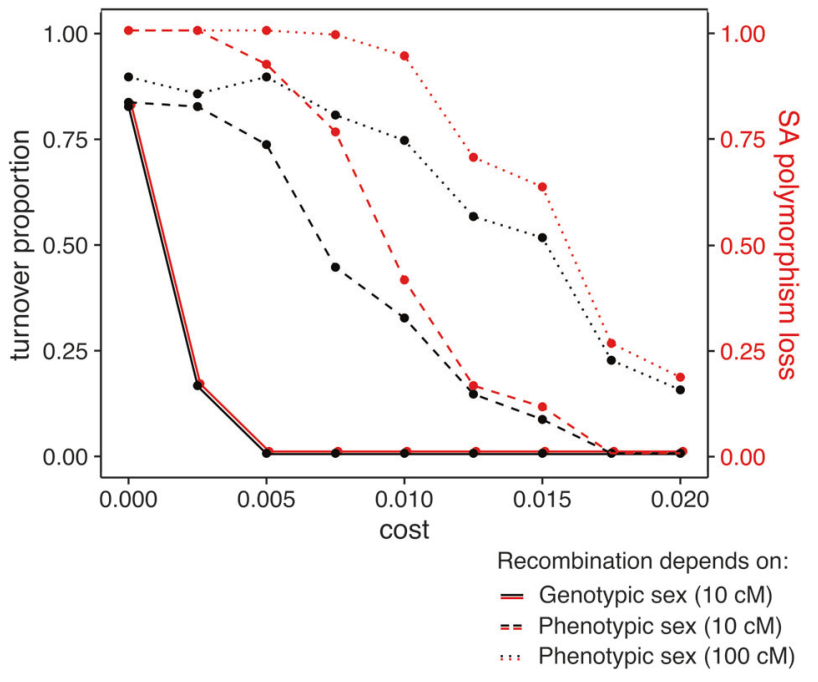

Fig. 3 Turnover proportion (black lines) and proportion of simulations during which the polymorphism at the SA locus is lost (red lines), as a function of the strength of sexually antagonistic selection (cost $c$ ), across 100 replicates. Parameter values: $\left\{N_{\mathrm{e}}=10^{3}, h=s=0\right\}$, the values in parentheses in the legend give the distance between the sexdetermining locus and the SA locus

results are consistent with classical SA theory results showing that SA polymorphism is more easily maintained with stronger SA selection (e.g., Kidwell et al. 1977; Jordan and Charlesworth 2012). Interestingly, mean SA allele fixation time is always shorter than the mean time to turnover, and turnovers in these simulations tend to closely follow the loss of polymorphism at the SA locus (Supplementary Figs. S8 and S9). This suggests that turnovers are favored by this loss of polymorphism. Rerunning simulations in which either the male beneficial-female detrimental or female beneficial-male detrimental allele is initially fixed on both the $\mathrm{X}$ and the $\mathrm{Y}$ chromosomes, brings turnover likelihood back to the neutral expectations (absence of SA locus), regardless of (i) which allele is fixed, and (ii) the value of $c$ (Supplementary Fig. S10). This confirms that fixation of either SA allele, made possible by recombination between the sex-determining locus and SA locus in $X Y f F$ females, is what increases turnover probability. This loss of polymorphism is likely mediated by genetic drift, as supported by the facts that (i) loss occurs less frequently at high selection coefficients, and (ii) the fixation probability of each of the two alleles is close to their initial frequencies (Supplementary Fig. S11). Thus, although SA variation on the ancestral sex-chromosome pair hinders transitions between male and female heterogamety, $\mathrm{X}-\mathrm{Y}$ recombination in $X Y f F$ females can break the association between $\mathrm{X}$ and $\mathrm{Y}$ chromosomes and female- or male-beneficial SA alleles. If either allele is lost by genetic drift, the transition probability increases, and goes back to the expected transition probability in the absence of a SA locus. 


\section{Comparisons with $\mathrm{XY}$ to $\mathrm{XY}$ turnovers}

To better compare our results to those from Blaser et al. (2013), we re-ran some simulations replacing the autosomal feminizing mutant allele $F$ by an epistatically dominant masculinizing allele $M$ (note that the mode of recombination arrest cannot impact the spread of the latter, as no $X Y$ females are produced throughout $X Y$ to $X Y$ turnovers: males are $X Y m m$ or $X X m M$, and females all $X X \mathrm{~mm}$, see Table 1A in Bull and Charnov (1977)). As expected, in the absence of SA variants linked to the resident sexdetermining locus, $X Y$ to $X Y$ turnovers occur with a higher probability than $\mathrm{XY}$ to $\mathrm{ZW}$ ones, especially if recombination depends on genotypic sex (Supplementary Fig. S12). In the presence of SA selection, this difference is exacerbated for $\log _{10}\left(N_{\mathrm{e}} h s\right)$ values ranging 0 to 1 (Supplementary Fig. S12), and increases with the selective cost of the SA alleles (Supplementary Fig. S13).

\section{Discussion}

As shown by Blaser et al. $(2013,2014)$ in a maleheterogametic sex-determining system, the load of deleterious mutations accumulating on the non-recombining $\mathrm{Y}$ chromosome can promote the spread of an emergent male sex-determining mutation, thereby causing a transition in the sex-determination mechanism: an $\mathrm{XY}$ to $\mathrm{XY}$ turnover. In sharp contrast, our present results show that this $\mathrm{Y}$ chromosome mutation load cannot favor the spread of an epistatically dominant female sex-determining mutation, and therefore does not favor $\mathrm{XY}$ to $\mathrm{ZW}$ turnovers. Depending on the mechanism that controls recombination arrest, a transition to a $\mathrm{ZW}$ system can either be strongly counter-selected, or just allowed to spread at a near neutral rate. On the one hand, if recombination arrest depends on genotypic sex (e.g., caused by a chromosomal inversion on the Y), mutations that fall within the interval $N_{\mathrm{e}} s=1$ and $N_{\mathrm{e}} h s=10$ strongly hinder turnovers (Fig. 2 and Supplementary Fig. S3, top left panels). This corresponds to the interval in which purifying selection is too weak to prevent fixation of deleterious mutations on the Y (Supplementary Fig. S1), but strong enough to prevent their fixation on the $\mathrm{X}$ (Supplementary Fig. S2). As thoroughly discussed in Blaser et al. (2013) $N_{\mathrm{e}} h s$ values that facilitate the accumulation of mutations on the $\mathrm{Y}\left(1<N_{\mathrm{e}} h s<10\right)$ are likely to be met in nature in many vertebrates species, and it has been confirmed empirically that non-recombining $\mathrm{Y}$ chromosomes tend to accumulate deleterious mutations at a faster rate than their X counterpart (e.g., Berlin and Ellegren 2006; Bergero and Charlesworth 2011; Zhou and Bachtrog 2012), providing the right conditions for Y-deleterious load to have an impact on the spread of sex determiners. On the other hand, if recombination arrest depends on phenotypic sex (e.g., in link with a genome-wide restriction of recombination in males), the load of deleterious mutations on the $\mathrm{Y}$ has far less impact on transitions (Fig. 2 and Supplementary Fig. S3, top right panels). The reason for this difference is that an $\mathrm{XY}$ to $\mathrm{ZW}$ turnover driven by an epistatically dominant mutation requires fixation of the $\mathrm{Y}$ chromosome as an autosome, which is costly if it is loaded with deleterious mutations. However, along the process, XY Ff females are produced, providing an opportunity for $\mathrm{X}-\mathrm{Y}$ recombination, and therefore purge of the $\mathrm{Y}$-deleterious load, but only if recombination depends on phenotypic sex. Our results also suggest that this opportunity for recombination can limit the negative effect that SA selection on resident sex-chromosome has on turnovers (Fig. 2 middle and bottom panels). Indeed, if recombination depends on genotypic sex, even the weakest cost of bearing a malebeneficial allele on the $\mathrm{Y}(c=0.005)$ is enough to greatly limit the spread of female sex-determining mutants. If recombination depends on phenotypic sex, conversely, recombination in $X Y F f$ females allows decoupling alleles at the sex-determining locus and SA locus, which can lead to the loss of SA variation by genetic drift.

Note that our SA model is relatively basic: selection is symmetric (the cost of being homozygous for the detrimental allele is the same in males and females) and the effect of SA allele is additive. It might seem more plausible that SA alleles are partially dominant in the sex in which they are advantageous, which, according to analytical models, should increase the stability of SA polymorphism (Fry 2010). While this might decrease the likelihood of XY to $\mathrm{ZW}$ turnovers when recombination depends on phenotypic sex by making it harder to fix SA alleles, it does not affect our results qualitatively: SA polymorphism can be lost only in this case, and not if recombination depends on genotypic sex. The impact of SA genes on transitions between male and female heterogamety has been studied in depth by van Doorn and Kirkpatrick (2010), who evaluate the effect of the presence of multiple SA alleles on both the ancestral and emergent pairs of sex chromosomes. They show that SA selection can trigger a XY to ZW transition if the selective advantage provided by linkage of female beneficial alleles to the emergent feminizing sexdetermining allele outweighs the cost of selection acting at the SA loci linked to the ancestral sex-determining locus. They also show that the strength of genetic linkage between SA and sex-determining loci has a strong impact on promoting or preventing turnovers: e.g., tighter linkage between the sex locus and SA locus on the ancestral sex chromosomes makes transitions more difficult. This parallels our findings on the impact of the mode of recombination suppression on turnovers: if recombination depends on phenotypic sex, recombination in sex-reversed XY females 
reduces linkage between the two loci, making turnovers more likely. Thus, according to our results, recombination depending on phenotypic sex would facilitate SA-driven transitions investigated by van Doorn and Kirkpatrick (2010), as recombination in XY females would lower linkage between the sex-determining and SA loci on the ancestral sex-chromosome pair.

The direct comparison of $\mathrm{XY}$ to $\mathrm{ZW}$ and $\mathrm{XY}$ to $\mathrm{XY}$ turnover likelihood in our models (Supplementary Fig. S12) suggests that if the sex-chromosome deleterious load plays a predominant role in sex-chromosome turnovers, transitions that maintain the heterogamety pattern should be more frequent than transitions along which the heterogametic sex switches, especially if recombination suppression depends on genotypic sex (and assuming mutant male and female determiners emerge at the same rate). It is worth noting, also, that in the absence of deleterious mutations, a SA polymorphism on the resident sex chromosomes has the potential to favor $\mathrm{XY}$ to $\mathrm{ZW}$ transitions over $\mathrm{XY}$ to $\mathrm{XY}$ ones, provided recombination depends on phenotypic sex (Supplementary Fig. S13). This arises because such a polymorphism, which normally hinders transitions, can be lost through $\mathrm{X}-\mathrm{Y}$ recombination in $\mathrm{XY} F f$ females, generated only in transitions between male and female heterogamety. These observations suggest that it might be possible to assess the relative impact of different evolutionary forces on sex-chromosome turnovers (e.g., SA selection vs. deleterious load) by examining the rates of different types of transitions across phylogenies in taxa that experience frequent sex-chromosome turnovers. For instance, an unprecedented rate of sex-chromosome turnovers has recently been described in ranid frogs (Jeffries et al. 2018), and most turnovers have preserved the ancestral mode of male heterogamety, which was interpreted as supporting a role for the load of deleterious mutations (as opposed to SA selection) in driving turnovers. As our present results show, however, this load should not entirely prevent transitions to female heterogamety, given that the lack of XY recombination is controlled by phenotypic sex in Ranidae (Rodrigues et al. 2018). Thus, a role for SA genes cannot be excluded here: as our results also show, turnovers are still much more likely to maintain male heterogamety under the range of deleterious mutations considered (i.e., between $N_{\mathrm{e}} s=1$ and $N_{\mathrm{e}} h s=10$ ) in the presence of male-beneficial alleles on the ancestral $\mathrm{Y}$ chromosome (Supplementary Fig. S13). Alternatively, given the genome-wide restriction of recombination in males, an autosomal male-beneficial mutation might be more likely to attract a new sex-determining gene than a female-beneficial mutation, since the new sex determiner will then immediately benefit from full linkage with the SA gene (Sardell et al. 2018). Turnovers towards systems where the sex showing reduced recombination is the heterogametic one might thereby be favored by SA selection, regardless of whether they involve a change in the heterogamety or not. Whether SA selection also plays a role in the sexchromosome turnovers of Ranidae requires further investigation. The rate of characterization of sex-chromosome systems has drastically increased in the last few years thanks to the development of genomic technologies, revealing that many taxa experience frequent changes in sex-determination mode (Gamble et al. 2015; Blackmon et al. 2017; Gammerdinger and Kocher 2018; Pennell et al. 2018). The record is nevertheless still too scarce in most groups to quantify the rates of different types of turnovers, but as data keep accumulating, it might become possible in the near future to test more thoroughly our hypotheses.

So far, we have only discussed sex-chromosome turnovers driven by epistatically dominant autosomal mutations, occurring in an initially male-heterogametic system. Our results can be readily expanded to other types of turnovers. Firstly, the system is symmetric regarding the reverse $\mathrm{ZW}$ to $X Y$ transition. The same mechanism would allow purging the $\mathrm{W}$ from its load of deleterious mutations, provided recombination is arrested in females, not in males (as documented in Lepidoptera). Secondly, the mechanism would also apply to so-called "homologous" turnovers, where the sex-determining mutation arises on the resident sex-chromosome pair, not on an autosome (as documented e.g., in Glandirana rugosa; Miura 2008). A mutation on the $\mathrm{X}$ making it a dominant female-determining sex-chromosome (W), for instance, would produce WY females in which $\mathrm{Y}$ can recombine and be purged before its fixation as a $\mathrm{Z}$ chromosome. In contrast, a mutation on the $\mathrm{X}$ making it a dominant male-determining allele (a new $\mathrm{Y}$ ) would induce a homologous $X Y$ to $X Y$ turnover during which the ancestral $\mathrm{Y}$ chromosome is eliminated and no $\mathrm{XY}$ females are produced (Bull and Charnov 1977). Therefore, homologous turnovers that either change or maintain the ancestral pattern of heterogamety should be impacted in a similar manner to their non-homologous counterparts by the accumulation of deleterious mutations and SA selection. Thirdly, transitions may occur through the spread of mutations that are not epistatically dominant over the resident sex determiner (Bull and Charnov 1977). For instance, a transition from male to female heterogamety can be caused by the spread of a "weakly male-determining mutation" $M$ in an originally male-heterogametic system (i.e., from $X X m m / X Y m m$ to $X X m M / X X M M$; case $2 \mathrm{~B}$ in Bull and Charnov 1977). No $X Y$ females are produced throughout this kind of transition, and the resident $\mathrm{Y}$ chromosome is the chromosome lost once the transition is complete. Hence, this suggests that a transition from male to female heterogamety can actually be favored by mutationload selection, but only if driven by a non-dominant maledetermining mutation. Conversely, a transition that 
maintains the pattern of heterogamety can be driven by the spread of a "mildly feminizing determining allele" $F$ in an originally male-heterogametic system (i.e., from $X X$ ffl $X Y$ ff to $Y Y F F / Y Y$ fF; cases $3 \mathrm{~B} 1$ and $3 \mathrm{~B} 2$ in Bull and Charnov 1977). Throughout these turnovers, $X Y$ females are produced, causing the ancestral $\mathrm{Y}$ to be purged from deleterious mutations before it goes to fixation as an autosome. Such an XY to XY turnover would not be favored (but not prevented either) by mutation-load selection. Therefore, the dominance relationship between sex-determining factors is also a parameter to be taken into account when considering the impact on sex-chromosome turnovers of deleterious mutations, SA polymorphism, and mode of recombination suppression.

A major finding of our study is that the mode of recombination suppression can have a crucial impact on the spread of sex-determining mutations. The ultimate and proximal causes of sex-chromosome recombination arrest are still poorly understood (Wright et al. 2016; Ponnikas et al. 2018), and although many sex chromosomes have been shown to carry a non-recombining region, it is still unknown how frequently recombination arrest depends on genotypic or phenotypic sex. Inversions are found on many non-recombining sex-chromosomes, and they have naturally been proposed as a potential cause for suppression, but empirical support remains sparse (Bergero and Charlesworth 2009), and it has been shown that inversions also tend to fix on sex chromosomes following the arrest (e.g., Silene latifolia, Bergero et al. 2008). Interestingly, sex-reversal experiments in a series of organisms, including houseflies (Inoue et al. 1983), crested newts (Wallace et al. 1997), medaka fish (Matsuda et al. 1999; Kondo et al. 2001) and tilapias (Campos-Ramos et al. 2009), clearly show that the patterns of recombination are essentially controlled by phenotypic sex (review in Perrin 2009). Hence, changes in heterogamety can certainly occur despite the accumulating load of deleterious mutations in a large range of organisms. However, this conclusion is likely to only apply at the early stages of sex-chromosome evolution. In the absence of turnovers, lack of recombination can lead over time to more profound changes, including evolution of structural differences (e.g., inversions), accumulation of transposable elements and many recessive deleterious mutations, and evolution of dosage compensation (Beukeboom and Perrin 2014). All these factors should contribute to prevent $X-Y$ recombination, and therefore, a direct change in heterogamety mediated by the spread of an epistatically dominant W mutation.

\section{Data archiving}

Data available from the Dryad Digital Repository: https:// doi.org/10.5061/dryad.5cc26s8.
Acknowledgements We are thankful to Jérôme Goudet and Elisa Cavoto for stimulating discussions and helpful comments, and Andrew Saunders for proofreading. The computations were performed at the Vital-IT (http://www.vital-it.ch) Center for high-performance computing of the SIB Swiss Institute of Bioinformatics. Funding was provided by the Swiss National Science Foundation (Grant Number 31003A_166323) to Nicolas Perrin.

\section{Compliance with ethical standards}

Conflict of interest The authors declare that they have no conflict of interest.

Publisher's note: Springer Nature remains neutral with regard to jurisdictional claims in published maps and institutional affiliations.

\section{References}

Bergero R, Charlesworth D (2009) The evolution of restricted recombination in sex chromosomes. Trends Ecol Evol 24:94-102

Bergero R, Charlesworth D (2011) Preservation of the Y transcriptome in a 10-million-year-old plant sex chromosome system. Curr Biol 21:1470-1474

Bergero R, Charlesworth D, Filatov DA, Moore RC (2008) Defining regions and rearrangements of the Silene latifolia $\mathrm{Y}$ chromosome. Genetics 178:2045-2053

Berlin S, Ellegren H (2006) Fast accumulation of nonsynonymous mutations on the female-specific $\mathrm{W}$ chromosome in birds. J Mol Evol 62:66-72

Beukeboom LW, Perrin N (2014). The evolution of sex determination. Oxford University Press, Oxford, UK

Blackmon H, Ross L, Bachtrog D (2017) Sex determination, sex chromosomes, and karyotype evolution in insects. J Hered 108:78-93

Blaser O, Grossen C, Neuenschwander S, Perrin N (2013) Sexchromosome turnovers induced by deleterious mutation load. Evolution 67:635-645

Blaser O, Neuenschwander S, Perrin N (2014) Sex-chromosome turnovers: the hot-potato model. Am Nat 183:140-146

Brelsford A, Rodrigues N, Perrin N (2016) High-density linkage maps fail to detect any genetic component to sex determination in a Rana temporaria family. J Evol Biol 29:220-225

Bull JJ, Charnov EL (1977) Changes in the heterogametic mechanism of sex determination. Heredity 39:1-14

Campos-Ramos R, Harvey SC, Penman DJ (2009) Sex-specific differences in the synaptonemal complex in the genus Oreochromis (Cichlidae). Genetica 135:325-332

Charlesworth D, Charlesworth B (1980) Sex differences in fitness and selection for centric fusions between sex-chromosomes and autosomes. Genet Res, Camb 35:205-214

Charlesworth D, Charlesworth B, Marais G (2005) Steps in the evolution of heteromorphic sex chromosomes. Heredity 95:118-128

van Doorn GS, Kirkpatrick M (2007) Turnover of sex chromosomes induced by sexual conflict. Nature 449:909-912

van Doorn GS, Kirkpatrick M (2010) Transitions between male and female heterogamety caused by sex-antagonistic selection. Genetics 186:629-645

Fisher RA (1931) The evolution of dominance. Biol Rev 6:345-368

Fry JD (2010) The genomic location of sexually antagonistic variation: some cautionary comments. Evolution 64:1510-1516

Gamble T, Coryell J, Ezaz T, Lynch J, Scantlebury DP, Zarkower D (2015) Restriction site-associated DNA sequencing (RAD-seq) reveals an extraordinary number of transitions among gecko sexdetermining systems. Mol Biol Evol 32:1296-1309 
Gammerdinger WJ, Kocher TD (2018) Unusual diversity of sex chromosomes in African cichlid fishes. Genes 9:480

Haldane JBS (1919) The combination of linkage values and the calculation of distances between the loci of linked factors. J Genet 8:299-309

Inoue H, Fukumori Y, Hiroyoshi T (1983) Mapping of autosomal male-determining factors of the housefly, Musca domestica L., by means of sex-reversal. Jpn J Genet 58:451-461

Jeffries DL, Lavanchy G, Sermier R, Sredl MJ, Miura I, Borzée A et al. (2018) A rapid rate of sex-chromosome turnover and nonrandom transitions in true frogs. Nat Commun 9:4088

Jordan CY, Charlesworth D (2012) The potential for sexually antagonistic polymorphism in different genome regions. Evolution 66:505-516

Kidwell JF, Clegg MT, Stewart FM, Prout T (1977) Regions of stable equilibria for models of differential selection in the two sexes under random mating. Genetics 85:171-183

Kirkpatrick M (2010) How and why chromosome inversions evolve. PLoS Biol 8:e1000501

Kondo M, Nagao E, Mitani H, Shima A (2001) Differences in recombination frequencies during female and male meioses of the sex chromosomes of the medaka, Oryzias latipes. Genet Res 78:23-30

Matsuda M, Sotoyama S, Hamaguchi S, Sakaizumi M (1999) Malespecific restriction of recombination frequency in the sex chromosomes of the medaka, Oryzias latipes. Genet Res 73:225-231

Miura I (2008) An evolutionary witness: The frog rana rugosa underwent change of heterogametic sex from $\mathrm{XY}$ male to $\mathrm{ZW}$ female. Sex Dev 1:323-331

Morgan TH (1914) No crossing over in the male of drosophila of genes in the second and third pairs of chromosomes. Bio Bull 26:195-204

Neuenschwander S, Michaud F, Goudet J (2018) quantiNemo 2: a swiss knife to simulate complex demographic and genetic scenarios, forward and backward in time. Bioinformatics 35:886-888

Pennell MW, Mank JE, Peichel CL (2018) Transitions in sex determination and sex chromosomes across vertebrate species. Mol Ecol 27:3950-3963

Perrin N (2009) Sex reversal: A fountain of youth for sex chromosomes? Evolution 63:3043-3049
Ponnikas S, Sigeman H, Abbott JK, Hansson B (2018) Why do sex chromosomes stop recombining? Trends Genet 34:492-503

Rice W (1984) Sex chromosomes and the evolution of sexual dimorphism. Evolution 38:735-742

Rice WR (1987) The accumulation of sexually antagonistic genes as a selective agent promoting the evolution of reduced recombination between primitive sex chromosomes. Evolution 41:911-914

Roberts RB, Ser RJ, Kocher TD (2009) Sexual conflict resolved by invasion of a novel sex determiner in Lake Malawi cichlid fishes. Science 326:998-1001

Rodrigues N, Betto-Colliard C, Jourdan-Pineau H, Perrin N (2013) Within-population polymorphism of sex-determination systems in the common frog (Rana temporaria). J Evol Biol 26:1569-1577

Rodrigues N, Studer T, Dufresnes C, Perrin N (2018) Sexchromosome recombination in common frogs brings water to the fountain-of-youth. J Evol Biol 35:942-948

Sardell JM, Cheng C, Dagilis AJ, Ishikawa A, Kitano J, Peichel CL et al. (2018) Sex differences in recombination in sticklebacks. G3 8:g3-200166

Saunders PA, Neuenschwander S, Perrin N (2018) Sex chromosome turnovers and genetic drift: A simulation study. J Evol Biol 31:1413-1419

Schartl M (2004) Sex chromosome evolution in non-mammalian vertebrates. Curr Opin Genet Dev 14:634-641

Tanaka Y (1914) A study of Mendelian factors in the Sikworm, Bombyx mori. Mol. Genet. Genomics. 12:161

Veller C, Muralidhar P, Constable GWA, Nowak MA (2017) Driftinduced selection between male and female heterogamety. Genetics 207:711-727

Volff JN, Nanda I, Schmid M, Schartl M (2007) Governing sex determination in fish: Regulatory putsches and ephemeral dictators. Sex Dev 1:85-99

Wallace H, Wallace BMN, Badawy GMI (1997) Lampbrush chromosomes and chiasmata of sex-reversed crested newts. Chromosoma 106:526-533

Wright AE, Dean R, Zimmer F, Mank JE (2016) How to make a sex chromosome. Nat Commun 7:1-8

Zhou Q, Bachtrog D (2012) Sex-specific adaptation drives early sex chromosome evolution in Drosophila. Science 337:341-345 